\title{
Sex differences in insulin resistance
}

Before menopause, women have a lower incidence of insulin resistance than men of a similar age. However, this protective effect disappears after menopause, with the incidence of insulin resistance and type 2 diabetes mellitus becoming similar in men and women, which suggests that oestrogen could have a protective effect. Now, new research indicates that $17 \beta$-oestradiol protects pro-opiomelanocortin (POMC) neurons from developing insulin resistance.

Previous work has shown that insulin excites POMC neurons in the hypothalamus by activating canonical transient receptor potential (TRPC) cation channels. "We hypothesized that females would be protected against central insulin resistance through preserving this vital signalling pathway in neurons, specifically the hypothalamic POMC neurons," explains corresponding author Martin Kelly.

Using electrophysiological techniques that enabled the measurement of TRPC channel activity, the researchers demonstrated that male mice with diet-induced obesity (DIO) exhibited reduced activation of TRPC channels in response to insulin, which led to reduced depolarization of POMC neurons. This effect was not seen in female mice with DIO; however, ovariectomized female mice with DIO responded similarly to the male mice, and the usual 'female' response was restored with replacement of $17 \beta$-oestradiol. The researchers also conducted single-cell RT-PCR to show that $17 \beta$-oestradiol increased the mRNA levels for the T-type calcium channel $\mathrm{Ca}_{v} 3.1$, which increased the excitability of POMC neurons, and downregulated Stim1 mRNA levels (STIM1 attenuates the coupling of insulin receptors to TRPC channels).

"Our results indicate that women are protected against central insulin resistance with DIO prior to menopause due to circulating oestrogens," concludes Kelly. The researchers have developed a non-steroidal oestrogen (STX), and are now planning to test whether STX protects against insulin resistance in a similar way to endogenous oestrogen.

\section{Claire Greenhill}

ORIGINAL ARTICLE Qiu, J. et al. Estradiol protects proopiomelanocortin neurons against insulin resistance. Endocrinology http://dx.doi.org/10.1210/ en.2017-00793 (2017)

FURTHER READING Morselli, E. et al. The effects of oestrogens and their receptors on

cardiometabolic health. Nat. Rev. Endocrinol. 13 , 352-364 (2017) 